\title{
Integrating tertiary Gleason pattern 5 into the ISUP grading system improves prediction of biochemical recurrence in radical prostatectomy patients
}

\author{
Masashi Kato ${ }^{1}$ - Akihiro Hirakawa ${ }^{2} \cdot$ Yumiko Kobayashi $^{3} \cdot$ Akiyuki Yamamoto $^{1} \cdot$ Ryo Ishida $^{4} \cdot$ Osamu Kamihira $^{5}$. \\ Tohru Kimura $^{6}$ - Tsuyoshi Majima ${ }^{1} \cdot$ Shohei Ishida ${ }^{1}$ - Yasuhito Funahashi ${ }^{1} \cdot$ Naoto Sassa $^{1} \cdot$ Takashi Fujita $^{1}$. \\ Yoshihisa Matsukawa ${ }^{1} \cdot$ Tokunori Yamamoto $^{1} \cdot$ Ryohei Hattori $^{7} \cdot$ Momokazu Gotoh $^{1} \cdot$ Toyonori Tsuzuki $^{8}$
}

Received: 20 May 2018 / Revised: 13 July 2018 / Accepted: 13 July 2018 / Published online: 4 September 2018

(c) United States \& Canadian Academy of Pathology 2018

\begin{abstract}
Although the presence of tertiary Gleason pattern 5 is reportedly related to unfavorable prostate cancer characteristics, few data are available regarding the effects of tertiary Gleason pattern 5 on the new ISUP (International Society of Urological Pathology) grading system in radical prostatectomy patients. In this study, we evaluated the effect of tertiary Gleason pattern 5 on biochemical recurrence following radical prostatectomy in patients with prostate cancer. We retrospectively evaluated 1000 patients with prostate cancer who underwent radical prostatectomy. The ISUP Grades were as follows: Grade 1, 16.3\%; Grade 2, 48.1\% (with tertiary Gleason pattern 5, 8.0\% and without tertiary Gleason pattern 5, 40.1\%); Grade 3, 21.9\% (with tertiary Gleason pattern 5, 9.1\% and without tertiary Gleason pattern 5, 12.8\%); Grade 4, 3.9\%; and Grade 5, 9.8\%. Biochemical recurrence-free survival differed significantly among the five groups (Grade $1-5)(P<0.0001)$. Grade 2 with tertiary Gleason pattern 5 had a significantly worse prognosis than Grade 2 without tertiary Gleason pattern 5. Similarly, Grade 3 with tertiary Gleason pattern 5 demonstrated a significantly worse prognosis than Grade 3 without tertiary Gleason pattern 5. When Grade 2 and Grade 3 were divided with or without tertiary Gleason pattern 5, the survival curves differed significantly among Grade 1, Grade 2 without tertiary Gleason pattern 5, Grade 2 with tertiary Gleason pattern 5, Grade 3 without tertiary Gleason pattern 5, Grade 3 with tertiary Gleason pattern 5, and Grade $4(P<0.0001)$ (hazard ratios: $1,1.14$, $1.86,2.23,3.53$, and 6.30). In a multivariate analysis, integrating tertiary Gleason pattern 5 into the ISUP Grade, PSA, and surgical margin status significantly predicted biochemical recurrence $(P<0.0001)$. Integrating tertiary Gleason pattern 5 into the ISUP grading system will improve the accuracy of patient outcome prediction following radical prostatectomy in patients with prostate cancer.
\end{abstract}

Electronic supplementary material The online version of this article (https://doi.org/10.1038/s41379-018-0121-8) contains supplementary material, which is available to authorized users.

\footnotetext{
Toyonori Tsuzuki

tsuzuki@aichi-med-u.ac.jp

1 Department of Urology, Nagoya University Graduate School of Medicine, Nagoya, Japan

2 Department of Biostatistics and Bioinformatics, Graduate School of Medicine, The University of Tokyo, Tokyo, Japan

3 Statistical Analysis Section, Center for Advanced Medicine and Clinical Research, Nagoya University Hospital, Nagoya, Japan

4 Department of Urology, Japanese Red Cross Nagoya Daini
}

\section{Introduction}

Gleason score is one the most important prognostic factors for prostate cancer. However, the original Gleason score has several issues to be resolved. For example, Gleason score 7 cases involve heterologous disease entities, and high-grade

\section{Hospital, Nagoya, Japan}

5 Department of Urology, Komaki City Hospital, Komaki, Aichi, Japan

6 Department of Urology, JCHO Chukyo Hospital, Nagoya, Japan

7 Department of Urology, Japanese Red Cross Nagoya Daiichi Hospital, Nagoya, Japan

8 Department of Surgical Pathology, School of Medicine, Aichi Medical University, Nagakute, Japan 
Gleason score cases, such as Gleason scores 9 and 10, have the similar clinical outcome [1]. The 2014 International Society of Urological Pathology (ISUP) consensus conference yielded a new grade group, ISUP grading system, to solve these issues, and subsequently, 2016 World Health Organization (WHO) classification has endorsed it [1, 2]. The Gleason score is now divided into five groups, and Gleason score 7 is divided into two categories: ISUP Grade 2 (Gleason score $3+4=7$ ) and ISUP Grade 3 (Gleason score $4+3=7)$. The ISUP grading system has been validated only for radical prostatectomy specimens.

The ISUP grading system proposes replacing the term tertiary-grade pattern into a minor high-grade pattern and using it for cases with minor and limited extent instead of just the third most common pattern. Finally, minor highgrade patterns will not change the ISUP Grade [3].

Pan et al. found that the presence of tertiary Gleason pattern 5 was a worse prognostic factor in radical prostatectomy cases and proposed reporting the presence of tertiary Gleason pattern 5 [4]. Later, Trock et al. found the presence of tertiary Gleason pattern 5 was related to high pathological stage and was an independent prognostic factor for biochemical recurrence in radical prostatectomy cases [5]. These reports were based on pre-2005 or 2005 ISUP grading criteria.

Few data are available on whether the presence of tertiary Gleason pattern 5 influences biochemical recurrence in radical prostatectomy patients. Therefore, in this study, we evaluated the effect of tertiary Gleason pattern 5 on recurrence following radical prostatectomy in patients with prostate cancer.

\section{Materials and methods}

We retrospectively evaluated 1020 patients treated with radical prostatectomy from 2005 to 2013 at Nagoya University Hospital, Japanese Red Cross Nagoya Daini Hospital, JCHO Chukyo Hospital, and Komaki City Hospital. All patients had been diagnosed with localized prostate cancer and had undergone radical prostatectomy. After excluding the patients with missing data or slides, 1000 patients were enrolled in this study. Practice on a range of lymphadenectomy and the operative approach (open, laparoscopic, or robotic) were decided by each institution. The clinical T (cT) of each tumor was re-assessed based on the 2009 UICC TNM classification system [6]. All resected specimens were serially sliced with 3 or $5 \mathrm{~mm}$ interval and totally embedded. All prostatectomy specimen slides were reviewed by a single genitourinary pathologist (TT) according to ISUP 2014. The tertiary Gleason pattern 5 was defined to be just the third most common pattern. The percentage of tertiary Gleason pattern 5 was ranged between
1 and $10 \%$ of the tumor in this study, although most of them were ranged within 5\% (167/172 cases).

Complete baseline and follow-up data were available for all 1000 patients. PSA was measured every 3 months over the 2 years following surgery and every 6 months thereafter. Biochemical recurrence following radical prostatectomy was defined according to the European Association of Urology guideline of rising PSA level $>0.2 \mathrm{ng} / \mathrm{ml}$ [7]. Clinical progression was defined as verification of local recurrence, distant metastasis, and/or newly diagnosed lymph node metastasis by imaging studies. The primary endpoint of this study was the biochemical recurrence-free survival, which was defined as the time from prostatectomy to biochemical recurrence. Cumulative incidence curves were used in a competing-risks setting to calculate the probabilities of biochemical recurrence, treating clinical progression and death as competing risks [8]. The cumulative incidence curves for the biochemical recurrence-free survival among each group were compared using Gray's test [9]. The influence of prognostic factors for biochemical recurrence-free survival was evaluated by using the Fine and Gray's model [10]. Data on age, PSA level at diagnosis, clinical and pathological $\mathrm{T}$ stage, the presence of Gleason pattern 5, and surgical margin status were analyzed to predict PSA recurrence after prostatectomy. A $P$ value $<0.05$ was considered statistically significant. All statistical analyses were performed using the SAS software (ver. 9.3; SAS Institute, Cary, NC, USA). This retrospective study was approved by the Institutional Review Board of Nagoya University Graduate School of Medicine.

\section{Results}

Table 1 lists the patient demographics. Median age at diagnosis was 67 years (range 45-80 years). The median serum prostate-specific antigen (PSA) level was $6.9 \mathrm{ng} / \mathrm{ml}$ (range $0.4-82 \mathrm{ng} / \mathrm{ml}$ ). The median follow-up period was 69 months (range 0.7-135 months). Pathological T (pT) occurred in 193 cases of pT2a, 110 of pT2b, 424 of pT2c, 233 of pT3a, and 40 of pT3b (Table 1). Overall, 262 patients $(26.2 \%)$ had PSA-progression and $14(1.4 \%)$ patients had clinical disease progression; one patient died of the disease $(0.1 \%)$, and $32(3.2 \%)$ patients died of other causes during the follow-up.

The number of patients in each grade was as follows: ISUP Grade 1, $16.3 \%(n=163)$; ISUP Grade 2, 48.1\% ( $n$ $=481$ ) (ISUP Grade 2 with tertiary Gleason pattern 5, 8.0\% $(n=80)$ and ISUP Grade 2 without tertiary Gleason pattern $5,40.1 \%(n=401))$; ISUP Grade 3, $21.9 \%(n=219)$ (ISUP Grade 3 with tertiary Gleason pattern $5,9.1 \%(n=91)$ and ISUP Grade 3 without tertiary Gleason pattern 5, $12.8 \%$ $(n=128))$; ISUP Grade $4,3.9 \%(n=39)$; and ISUP Grade 
Table 1 Clinical and pathological characteristics $(n=1000)$

\begin{tabular}{|c|c|c|c|c|c|c|}
\hline ISUP Grade & & $\begin{array}{l}1 \\
n=163\end{array}$ & $\begin{array}{l}2 \\
n=481\end{array}$ & $\begin{array}{l}3 \\
n=219\end{array}$ & $\begin{array}{l}4 \\
n=39\end{array}$ & $\begin{array}{l}5 \\
n=98\end{array}$ \\
\hline Age & & $\begin{array}{l}67 \\
(46-77)\end{array}$ & $\begin{array}{l}66 \\
(45-79)\end{array}$ & $\begin{array}{l}68 \\
(50-80)\end{array}$ & $\begin{array}{l}67 \\
(56-79)\end{array}$ & $\begin{array}{l}69 \\
(48-77)\end{array}$ \\
\hline Follow-up period & Months & $\begin{array}{l}77 \\
(4.9-135)\end{array}$ & $\begin{array}{l}68.4 \\
(0.7-132)\end{array}$ & $\begin{array}{l}67.9 \\
(4.6-129)\end{array}$ & $\begin{array}{l}83 \\
(6.6-122)\end{array}$ & $\begin{array}{l}64.5 \\
(16.6-121)\end{array}$ \\
\hline PSA & $\mathrm{ng} / \mathrm{ml}$ & $\begin{array}{l}5.7 \\
(1.1-19.5)\end{array}$ & $\begin{array}{l}6.5 \\
(0.4-50.9)\end{array}$ & $\begin{array}{l}8 \\
(3.1-81)\end{array}$ & $\begin{array}{l}7 \\
(2.5-17)\end{array}$ & $\begin{array}{l}8.3 \\
(4-82)\end{array}$ \\
\hline Clinical $\mathrm{T}$ & $\begin{array}{l}\text { cT1c, cT2 } \\
\text { cT3a } \\
\text { cT3b }\end{array}$ & 163 & $\begin{array}{l}467 \\
14\end{array}$ & $\begin{array}{l}212 \\
6 \\
1\end{array}$ & $\begin{array}{l}36 \\
2 \\
1\end{array}$ & $\begin{array}{l}93 \\
3 \\
2\end{array}$ \\
\hline Pathological T & $\begin{array}{l}\text { pT2a } \\
\text { pT2b } \\
\text { pT2c } \\
\text { pT3a } \\
\text { pT3b }\end{array}$ & $\begin{array}{l}70 \\
20 \\
71 \\
2\end{array}$ & $\begin{array}{l}78 \\
55 \\
244 \\
97 \\
7\end{array}$ & $\begin{array}{l}32 \\
25 \\
69 \\
80 \\
13\end{array}$ & $\begin{array}{l}6 \\
5 \\
13 \\
12 \\
3\end{array}$ & $\begin{array}{l}7 \\
5 \\
27 \\
42 \\
17\end{array}$ \\
\hline $\begin{array}{l}\text { Margin status } \\
\text { Tertiary Gleason pattern } 5\end{array}$ & Positive & $\begin{array}{l}25(15 \%) \\
0 / 163 \\
(0 \%)\end{array}$ & $\begin{array}{l}193(40 \%) \\
80 / 481 \\
(17 \%)\end{array}$ & $\begin{array}{l}108(49 \%) \\
91 / 219 \\
(42 \%)\end{array}$ & $\begin{array}{l}9(24 \%) \\
1 / 39 \\
(3 \%)\end{array}$ & $44(44 \%)$ \\
\hline
\end{tabular}

5, 9.8\% ( $n=98)$. Only one patient classified as ISUP Grade 4 with tertiary Gleason pattern 5 was categorized into ISUP Grade 5 because this is a minor population and usually has a poor prognosis.

PSA progression-free survival differed significantly among the five groups (ISUP Grades $1-5) \quad(P<0.0001)$ (Fig. 1, Table 2). In ISUP Grade 2 and ISUP Grade 3, margin status, PSA, and tertiary Gleason pattern 5 were significantly able to predict the prognosis (Table 3 ).

ISUP Grade 2 with tertiary Gleason pattern 5 had a significantly worse prognosis than ISUP Grade 2 without tertiary Gleason pattern 5. Similarly, ISUP Grade 3 with tertiary Gleason pattern 5 had a significantly worse prognosis than ISUP Grade 3 without tertiary Gleason pattern 5 (Table 4). In contrast, ISUP Grade 2 with tertiary Gleason pattern 5 and whole ISUP Grade 3, and ISUP Grade 3 with tertiary Gleason pattern 5 and whole ISUP Grade 4 had the same prognosis $(P=0.333$ and $P=0.129$ ) (Supplement 1, 2).

When ISUP Grade 2 and ISUP Grade 3 were divided with or without tertiary Gleason pattern 5, the survival curves differed significantly among ISUP Grade 1, ISUP Grade 2 without tertiary Gleason pattern 5, ISUP Grade 2 with tertiary Gleason pattern 5, ISUP Grade 3 without tertiary Gleason pattern 5, ISUP Grade 4, and ISUP Grade $5(P<0.0001)$ (hazard ratios; $1,1.14,1.86,2.23,3.53,6.30$, and 5.62) (Fig. 2). In a multivariate analysis, integrating tertiary Gleason pattern 5 into ISUP Grade, PSA level at diagnosis, and surgical margin status significantly predicted the prognosis $(P<$ $0.0001,<0.0001$ and $<0.0001$ respectively) (Table 4 ).

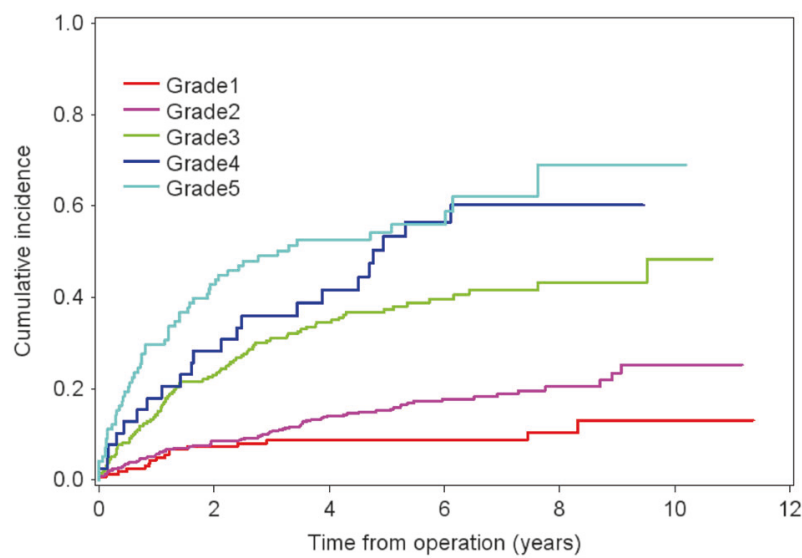

Fig. 1 Cumulative incidence curves for prostate-specific antigen (PSA) progressive survival after prostatectomy on the ISUP grading system $(P<0.0001)$

\section{Discussion}

The presence of tertiary Gleason pattern 5 is a well-known adverse prognostic factor in patients who undergo a radical prostatectomy [5, 11, 12]. The ISUP 2014 and 2016 WHO classifications recommend that minor high-grade patterns should not change the ISUP Grade [3]. However, this method using syntax seems to be less useful for urologists because tertiary Gleason pattern 5 seems to have a minor impact on patient outcome. A recent study concluded that this tertiary Gleason pattern 5 becomes stronger at Gleason score (GS) $3+4$ and $4+3$ cases but not for GS $4+4$ cases 
Table 2 Biochemical recurrence-free survival and variables in ISUP Grade $(n=1000)$

\begin{tabular}{|c|c|c|c|c|c|}
\hline \multicolumn{6}{|l|}{ Multivariate } \\
\hline Variables & Category & $\begin{array}{l}\text { Hazard } \\
\text { ratio }\end{array}$ & $\begin{array}{l}95 \% \\
\text { Lower }\end{array}$ & $\begin{array}{l}95 \% \\
\text { Upper }\end{array}$ & $P$ value \\
\hline \multirow[t]{5}{*}{ ISUP Grade } & Grade 1 & 1 & & & \\
\hline & Grade 2 & 1.24 & 0.719 & 2.139 & 0.439 \\
\hline & Grade 3 & 2.68 & 1.53 & 4.694 & 0.0006 \\
\hline & Grade 4 & 6.118 & 3.246 & 11.532 & $<0.0001$ \\
\hline & Grade 5 & 5.324 & 2.937 & 9.652 & $<0.0001$ \\
\hline \multirow[t]{2}{*}{ Age } & $65>$ & 1 & & & \\
\hline & $65 \leqq$ & 1.03 & 0.781 & 1.358 & 0.8353 \\
\hline \multirow[t]{2}{*}{ Clinical T } & cT2 & 1 & & & \\
\hline & cT3 & 0.881 & 0.458 & 1.696 & 0.7048 \\
\hline \multirow[t]{2}{*}{ Pathological T } & pT2 & 1 & & & \\
\hline & pT3 & 1.315 & 0.989 & 1.748 & 0.0597 \\
\hline \multirow[t]{2}{*}{ Margin status } & Negative & 1 & & & \\
\hline & Positive & 3.135 & 2.351 & 4.181 & $<0.0001$ \\
\hline PSA & Continuous & 1.027 & 1.017 & 1.037 & $<0.0001$ \\
\hline
\end{tabular}

Table 3 Biochemical relapse-free survival and variables in ISUP Grades 2 and $3(n=700)$

\begin{tabular}{|c|c|c|c|c|c|}
\hline \multicolumn{6}{|l|}{ Multivariate } \\
\hline Variables & Category & $\begin{array}{l}\text { Hazard } \\
\text { ratio }\end{array}$ & $\begin{array}{l}95 \% \\
\text { Lower }\end{array}$ & $\begin{array}{l}95 \% \\
\text { Upper }\end{array}$ & $P$ value \\
\hline \multirow[t]{2}{*}{ ISUP Grade } & Grade 2 & 1 & & & \\
\hline & Grade 3 & 1.946 & 1.404 & 2.697 & $<0.0001$ \\
\hline \multirow[t]{2}{*}{ Age } & $65>$ & 1 & & & \\
\hline & $65 \leqq$ & 0.976 & 0.696 & 1.368 & 0.8874 \\
\hline \multirow[t]{2}{*}{ Clinical $\mathrm{T}$} & cT2 & 1 & & & \\
\hline & cT3 & 1.227 & 0.589 & 2.556 & 0.5843 \\
\hline \multirow[t]{2}{*}{ Pathological T } & pT2 & 1 & & & \\
\hline & pT3 & 1.093 & 0.772 & 1.549 & 0.6149 \\
\hline \multirow[t]{2}{*}{ Margin status } & Negative & 1 & & & \\
\hline & Positive & 3.484 & 2.348 & 4.978 & $<0.0001$ \\
\hline PSA & $\begin{array}{l}\text { Continuous } \\
\text { Variables }\end{array}$ & 1.029 & 1.017 & 1.041 & $<0.0001$ \\
\hline \multirow{2}{*}{$\begin{array}{l}\text { Tertiary } \\
\text { Gleason } \\
\text { pattern } 5\end{array}$} & Absent & 1 & & & \\
\hline & Present & 1.63 & 1.156 & 2.296 & 0.0053 \\
\hline
\end{tabular}

[13]. Ozsoy et al. reported that upgrading the grade group by one grade when tertiary Gleason pattern 5 is present would more accurately predict the risk of biochemical recurrence for GS 7 [14]. Lucca et al. also reported that the risk of tertiary Gleason pattern 5 seemed to be comparable between patients with tertiary Gleason pattern 5 and their counterparts in the next higher ISUP Grade without tertiary Gleason pattern 5 .

A potential adverse effect of tertiary Gleason pattern 5 was first reported by Pan et al. [4]. Later, other groups also reported that the presence of tertiary Gleason pattern 5 was as an independent predictor of treatment failure after prostatectomy [11, 12]. Some studies found that GS $3+4$ with tertiary Gleason pattern 5 had almost the same risk of biochemical recurrence as GS $4+3$. Subsequently, Trock et al. demonstrated that GS $4+3$ with tertiary Gleason pattern 5 behaved like GS $4+4$ [5]. In contrast, Lucca et al. reported on 4146 prostatectomy patients by various pathologists at five institutions and found that GS $3+4=7$ with tertiary Gleason pattern 5 was associated with a slightly higher risk of biochemical recurrence compared to GS $4+3=7$ [15]. Adam et al. reported that the presence of tertiary Gleason pattern 5 was an independent predictor of biochemical recurrence after prostatectomy with the most potent prognostic effect in GS $3+4$ and $4+3$ cases [16]. Because these reports were evaluated before the 2014 ISUP conference, no data are available regarding the significance of tertiary Gleason pattern 5 in the current classification system. After the conference, several reports suggested that $5 \%$ is the upper limit of tertiary Gleason pattern 5, although they did not provide objective data to verify their statement [17]. A recent study also demonstrated that the minimal Gleason pattern 5 component has a substantial prognostic effect in Gleason 7 carcinomas. The authors also proposed an integrated quantitative Gleason score [18]. Another study reported that the presence of tertiary Gleason pattern 5 was correlated with higher PSA levels, a higher rate of lymph node metastasis, positive surgical margins, extraprostatic extension, and seminal vesicle invasion when compared with patients in the same grade without tertiary Gleason pattern 5 .

It has been unclear to what degree tertiary Gleason pattern 5 can play a prognostic role. Initially, Pan et al. reported that tertiary Gleason pattern 5 occurs in very limited amounts in Gleason pattern 5 [4]. Some subsequent studies used a cut-off $<5 \%[15,19]$, and others reported no limit for the tertiary Gleason pattern 5 [16]. No definitive upper amount of tertiary Gleason pattern 5 and a minimal amount of Gleason pattern 5 has been proposed as leading to a significantly poor patient prognosis. Attendances at the 2014 ISUP consensus meeting did not vote for a specific cut-off value of $5 \%$ for tertiary Gleason pattern 5 [3]. The rationale for restricting the term tertiary to cases with $<5 \%$ of the Gleason pattern 5 component is to equate a tertiary pattern, not only the third most common pattern; however, this has not been confirmed in a large cohort study [20]. A recent report found that the presence of $<5 \%$ tertiary Gleason pattern 5 imparts an intermediate biochemical recurrence relative to the next highest grade in ISUP Grade 
Table 4 Biochemical recurrence-free survival and variables in ISUP Grade with integrating tertiary Gleason pattern $5(n=1000)$

\begin{tabular}{|c|c|c|c|c|c|}
\hline \multicolumn{6}{|l|}{ Multivariate } \\
\hline Variables & Category & Hazard ratio & 95\% Lower & $95 \%$ Upper & $P$ value \\
\hline \multirow[t]{7}{*}{ ISUP Grade } & Grade 1 & 1 & & & \\
\hline & $\begin{array}{l}\text { Grade } 2 \text { without tertiary Gleason } \\
\text { pattern } 5\end{array}$ & 1.142 & 0.654 & 1.994 & 0.6412 \\
\hline & Grade 2 with tertiary Gleason pattern 5 & 1.858 & 0.957 & 3.607 & 0.0672 \\
\hline & $\begin{array}{l}\text { Grade } 3 \text { without tertiary Gleason } \\
\text { pattern } 5\end{array}$ & 2.233 & 1.222 & 4.08 & 0.009 \\
\hline & Grade 3 with tertiary Gleason pattern 5 & 3.537 & 1.928 & 6.488 & $<0.0001$ \\
\hline & Grade 4 & 6.296 & 3.342 & 11.86 & $<0.0001$ \\
\hline & Grade 5 & 5.618 & 3.098 & 10.186 & $<0.0001$ \\
\hline \multirow[t]{2}{*}{ Age } & $65>$ & 1 & & & \\
\hline & $65 \leqq$ & 1.02 & 0.773 & 1.346 & 0.8877 \\
\hline \multirow[t]{2}{*}{ Clinical T } & $\mathrm{cT} 2$ & 1 & & & \\
\hline & cT3 & 0.946 & 0.504 & 1.776 & 0.8637 \\
\hline \multirow[t]{2}{*}{ Pathological T } & pT2 & 1 & & & \\
\hline & pT3 & 1.243 & 0.934 & 1.653 & 0.1358 \\
\hline \multirow[t]{2}{*}{ Margin status } & Negative & 1 & & & \\
\hline & Positive & 3.139 & 2.354 & 4.186 & $<0.0001$ \\
\hline PSA (ng/ml) & Continuous & 1.025 & 1.015 & 1.035 & $<0.0001$ \\
\hline
\end{tabular}

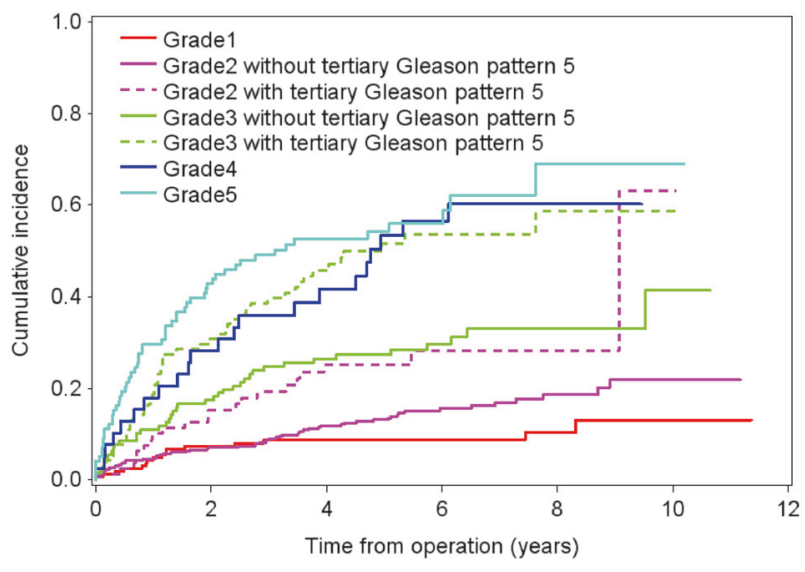

Fig. 2 Cumulative incidence curves for prostate-specific antigen (PSA) progressive survival after prostatectomy on the ISUP grading system integrating tertiary Gleason pattern $5(P<0.0001)$

2 and ISUP Grade 3 and could upgrade the category in ISUP Grade 4. In contrast, further expansion of tertiary Gleason pattern 5 has less of an effect on Gleason 7 carcinomas [18]. The definitive upper rate of tertiary Gleason pattern 5 seems to be controversial and will be elucidated in the future.

The present study had some limitations. First, the study population was small, and a nonrandomized retrospective design was used. Tertiary Gleason pattern 5 was found in $16.6 \%$ of ISUP Grade 2 (Gleason $3+4$ ) and $41.6 \%$ of ISUP Grade 3 (Gleason $4+3$ ). The reported prevalence of tertiary Gleason pattern 5 ranged to $50 \%$ [11, 21-26].
Therefore, our study population did not deviate from those of studies reported previously. Second, the number of ISUP Grade 4 and ISUP Grade 5 cases was relatively small compared with other grades. Because our institutions underwent neoadjuvant therapy at one time for high-risk and very high-risk patients, the number of enrolled patients was limited. Therefore, only limited ISUP Grade 5 cases regarded as clinically localized and better prognosis could have been enrolled in this cohort. The primary purpose of this study is to investigate the impact of incorporating Gleason tertiary pattern 5 on ISUP Grades 2 and 3, and we don't believe this selection bias for high ISUP Grade cases influences on a significant impact on our conclusions. Third, the surgical approach including lymph node dissection was not standardized in all cases. Nevertheless, our study had some advantages including an extended follow-up period (about 1.5-2 times longer than previously reported ones $[14,15,20])$ and one central pathologist who standardized the pathological evaluation of the radical prostatectomy specimens compared with recent reports, so upgrading tertiary Gleason pattern 5 in ISUP Grade 2 and ISUP Grade 3 is becoming more likely.

\section{Conclusions}

Integrating tertiary Gleason pattern 5 into the ISUP grading system will improve the accuracy of patient outcome predictions. ISUP Grade 2 and ISUP Grade 3 were upgraded 
by the presence of tertiary Gleason pattern 5 following radical prostatectomy in patients with prostate cancer.

\section{Compliance with ethical standards}

Conflict of interest The authors declare that they have no conflict of interest.

\section{References}

1. Epstein JI, Zelefsky MJ, Sjoberg DD, et al. A contemporary prostate cancer grading system: a validated alternative to the Gleason Score. Eur Urol. 2016;69:428-35.

2. Humphrey PA, Moch H, Cubilla AL, Ulbright TM, Reuter VE. The2016 WHO classification of tumours of the urinary system and male genital organs-Part B: prostate and bladder tumours. Eur Urol. 2016;70:106-19.

3. Epstein JI, Egevad L, Amin MB. et al. The 2014 International Society of Urological Pathology (ISUP) Consensus Conference on Gleason grading of prostatic carcinoma: definition of grading patterns and proposal for a new Grading System. Am J Surg Pathol. 2016;40:244-52.

4. Pan CC, Potter SR, Partin AW, Epstein JI. The prognostic significance of tertiary Gleason patterns of higher grade in radical prostatectomy specimens: a proposal to modify the Gleason grading system. Am J Surg Pathol. 2000;24:563-9.

5. Trock BJ, Guo CC, Gonzalgo ML, Magheli A, Loeb S, Epstein JI. Tertiary Gleason patterns and biochemical recurrence after prostatectomy: proposal for a modified Gleason scoring system. J Urol. 2009;182:1364-70.

6. Sobin LH, Gospodarowicz MK, Wittekind C. TNM Classification of Malignant Tumors. 7th ed. Oxford, UK: Wiley-Blackwell; 2009.

7. Cornford P, Bellmunt J, Bolla M, et al. EAU-ESTRO-SIOG guidelines on prostate cancer. Part II: treatment of relapsing, metastatic, and castration-resistant prostate cancer. Eur Urol. 2017;71:630-42.

8. Gooley TA, Leisenring W, Crowley J, Storer BE. Estimation of failure probabilities in the presence of competing risks: new representations of old estimators. Stat Med. 1999;18:695-706.

9. Gray RJ. A class of K-sample tests for comparing the cumulative incidence of a competing risk. Ann Stat. 1988;16:1141-54.

10. Fine JP, Gray RJ. A proportional hazards model for the subdistribution of a competing risk. J Am Stat Assoc. 1999;94:496-509.

11. Hattab EM, Koch MO, Eble JN, Lin H, Cheng L. Tertiary Gleason pattern 5 is a powerful predictor of biochemical relapse in patients with Gleason score 7 prostatic adenocarcinoma. J Urol. 2006;175:1695-9. discussion 1699.
12. van Oort IM, Schout BM, Kiemeney LA, Hulsbergen CA, Witjes JA. Does the tertiary Gleason pattern influence the PSA progression-free interval after retropubic radical prostatectomy for organ-confined prostate cancer? Eur Urol. 2005;48:572-6.

13. Mosse CA, Magi-Galluzzi C, Tsuzuki T, Epstein JI. The prognostic significance of tertiary Gleason pattern 5 in radical prostatectomy specimens. Am J Surg Pathol. 2004;28:394-8.

14. Jang WS, Yoon CY, Kim MS, et al. The prognostic role of tertiary Gleason pattern 5 in a contemporary grading system for prostate cancer. Prostate Cancer Prostatic Dis. 2017;20:93-98.

15. Lucca I, Shariat SF, Briganti A, et al. Validation of tertiary Gleason pattern 5 in Gleason score 7 prostate cancer as an independent predictor of biochemical recurrence and development of a prognostic model. Urol Oncol. 2015;33:71 e21-6.

16. Adam M, Hannah A, Budaus L, et al. A tertiary Gleason pattern in the prostatectomy specimen and its association with adverse outcome after radical prostatectomy. J Urol. 2014;192:97-101.

17. Baras AS, Nelson JB, Han M, Parwani AV, Epstein JI. The effect of limited (tertiary) Gleason pattern 5 on the new prostate cancer grade groups. Hum Pathol. 2017;63:27-32.

18. Sauter G, Steurer S, Clauditz TS, et al. Clinical utility of quantitative Gleason grading in prostate biopsies and prostatectomy specimens. Eur Urol. 2016;69:592-8.

19. Choy B, Pearce SM, Anderson BB, et al. Prognostic significance of percentage and architectural types of contemporary Gleason pattern 4 prostate cancer in radical prostatectomy. Am J Surg Pathol. 2016;40:1400-6.

20. Borhan W, Epstein JI. Significance of Gleason Score 7 with tertiary pattern 5 at radical prostatectomy. Urology. 2017;100:175-9.

21. Aihara M, Wheeler TM, Ohori M, Scardino PT. Heterogeneity of prostate cancer in radical prostatectomy specimens. Urology. 1994;43:60-6.discussion 66-7.

22. Epstein JI, Feng Z, Trock BJ, et al. Upgrading and downgrading of prostate cancer from biopsy to radical prostatectomy: incidence and predictive factors using the modified Gleason grading system and factoring in tertiary grades. Eur Urol. 2012;61:1019-24.

23. Gleason DF. Histologic grading of prostate cancer: a perspective. Hum Pathol. 1992;23:273-9.

24. Isbarn H, Ahyai SA, Chun FK, et al. Prevalence of a tertiary Gleason grade and its impact on adverse histopathologic parameters in a contemporary radical prostatectomy series. Eur Urol. 2009;55:394-401.

25. Ruijter ET, van de Kaa CA, Schalken JA, Debruyne FM, Ruiter DJ. Histological grade heterogeneity in multifocal prostate cancer. Biological and clinical implications. J Pathol. 1996;180:295-9.

26. Shah RB, Tadros Y. Adenocarcinoma of the prostate with Gleason pattern 5 on core biopsy: frequency of diagnosis, morphologic subpatterns, and relation to pattern distribution based on the modified Gleason grading system. Hum Pathol. 2014;45: 2263-9. 